\title{
Dynamic modeling and optimal control of cystic echinococcosis
}

\author{
Xinmiao Rong ${ }^{1,2}$, Meng Fan ${ }^{2 *}$, Huaiping Zhu ${ }^{3}$ and Yaohui Zheng ${ }^{4}$
}

\begin{abstract}
Background: Cystic echinococcosis is one of the most severe helminth zoonosis with a drastic impact on human health and livestock industry. Investigating optimal control strategy and assessing the crucial factors are essential for developing countermeasures to mitigate this disease.

Methods: Two compartment models were formulated to study the dynamics of cystic echinococcosis transmission, to evaluate the effectiveness of various control measures, and to find the optimal control strategy. Sensitive analyses were conducted by obtaining PRCCs and contour plot was used to evaluate the effect of key parameters on the basic reproduction number. Based on forward-backward sweep method, numerical simulations were employed to investigate effects of key factors on the transmission of cystic echinococcosis and to obtain the optimal control strategy.
\end{abstract}

Results: The food resources of stray dog and invalid sheep vaccination rate, which are always neglected, were significant to the transmission and control of cystic echinococcosis. Numerical simulations suggest that, the implementation of optimal control strategy can significantly reduce the infections. Improving the cost of health education and domestic dog deworming could not decrease human infections.

Conclusions: Our study showed that only a long-term use of the optimal control measures can eliminate the disease. Meanwhile, during the intervention, sheep vaccination and stray dogs disposing should be emphasized ahead of domestic dogs deworming to minimize the control cost. Simultaneously reducing other wild intermediate hosts and strengthening the sheep vaccination as well as disposing the stray dogs would be most effective.

Keywords: Cystic echinococcosis, Dynamic modeling, Optimal control, Global stability, Sheep vaccination

\section{Background}

Echinococcosis is an environmental driven zoonotic disease caused by eggs of Echinococcus transmitted from carnivores, which results in substantial morbidity and mortality in most areas of the world [1]. The latest estimation for the global burden of cystic echinococcosis (CE) was 184,000 new cases per annum resulting in 184,000 disability adjusted life years (DALYs), which led to a loss of 760 million dollars a year $[2,3]$. The global burden of alveolar echinococcosis (AE) was estimated to be 18,200

\footnotetext{
*Correspondence: mfan@nenu.edu.cn
}

2 School of Mathematics and Statistics, Northeast Normal University, 5268 Renmin Street, Changchun, Jilin 130024, People's Republic of China

Full list of author information is available at the end of the article cases per annum, resulting in approximately 666,000 DALYs, and the mortality of infectious humans without treatment may exceed $90 \%$ in $10-15$ years. Whilst, $91 \%$ of the human cases and 95\% of the DALYs were assessed to be in China $[4,5]$. Indeed, in western Sichuan, CE and AE have been shown to be highly endemic over 900,000 $\mathrm{km}^{2}$ [6]. Mass abdominal ultrasound screening result has revealed that the village prevalence rate of human CE was $12.1 \%$, while that of human AE was 14.3\% [7]. Studies of dogs showed that the dog prevalence was 14.5\% [8].

Some elimination exercises were based primarily on health education, praziquantel (PZQ)-based dogs deworming or abolition of individual slaughter of sheep, most successful control cases occurred on small islands $[1,9]$. As for Sichuan Province, the Ministry of Health original author(s) and the source, provide a link to the Creative Commons licence, and indicate if changes were made. The images or other third party material in this article are included in the article's Creative Commons licence, unless indicated otherwise in a credit line to the material. If material is not included in the article's Creative Commons licence and your intended use is not permitted by statutory regulation or exceeds the permitted use, you will need to obtain permission directly from the copyright holder. To view a copy of this licence, visit http://creativecommons.org/licenses/by/4.0/. The Creative Commons Public Domain Dedication waiver (http://creativeco mmons.org/publicdomain/zero/1.0/) applies to the data made available in this article, unless otherwise stated in a credit line to the data. 
of the People's Republic of China launched a plan in 2006 to address the main interventions involving dog deworming, stray dogs disposing, sheep vaccination programs, and health education [10]. However, the data collected from the studies of $\mathrm{He}$ et al. [11, 12] and Sichuan Province [13] showed that, despite the interventions described above, the number of CE human cases fluctuates from 2007 to 2011, and then increases between 2011 and 2016 (Fig. 1a). In addition, the trend of the infection rate of dogs is similar to that of human cases, fluctuating between 2007 and 2013, and increasing significantly between 2013 and 2016 (Fig. 1b triangle line). Yet, unlike the prevalence of humans and dogs, the infection ratio of sheep keeps declining in the years from 2007 to 2016 (Fig. 1b dot line).

Actually, there are several possible factors that cause abnormalities in humans and dogs as shown in the figure. First, due to the dispersed population and seasonal problems, it is difficult to achieve a standard schedule for monthly dogs deworming and stray dogs disposing [10]. The other is that time-independent sustainability of control implementation may not be sufficient to adapt to different environments [1]. At the same time, strong CE control interventions result in high costs. Then, we want to study the impact of stray dogs on the CE transmission and explore an optimal control strategy that can reduce both $\mathrm{CE}$ and costs. The observations in Fig. 1b indicate that sheep control is essentially effective, but the effectiveness of the vaccination program is rarely evaluated. Hence, we will also assess the contribution of sheep vaccination to the control of this endemic.
Some mathematical models have been developed to explain the transmission dynamics among human, dog and livestock, to assess control measures and to predict intervention outcomes in some Asian countries [2, 10, 14-16]. Yang et al. [15] used statistical analysis to conclude that a control program, which combined sheep vaccination and dog anthelmintic treatment, could achieve the goal of echinococcosis control in the long term. Moss et al. [16] considered the reinfection of canine echinococcosis to investigate the role of dogs in the spread of Echinococcus multilocularis in Tibetan communities of Sichuan Province. The results suggested that dog deworming could be an effective strategy to reduce the endemic in those communities. Craig et al. in [10] pointed out that combining treatment and control measures to control echinococcosis was the most effective potential.

The optimal control theory is a tool to find the optimal measures among comprehensive implementation interventions, which has been applied to many control for infectious disease [17-20], including the optimal risk management of human alveolar echinococcosis in Hokkaido [21]. Usually, the control of CE remains notoriously difficult, time-consuming and costly, especially in large scale campaign in remote and larger pastoral communities [1]. The prevention and control of CE require substantial financial resources. In order to explore the mechanism of CE transmission, to investigate the optimal control strategy, and to evaluate the effectiveness of vaccination program for sheep, we formulated a new dynamic model which develops the model proposed in [22] and incorporates into the sheep vaccination program.
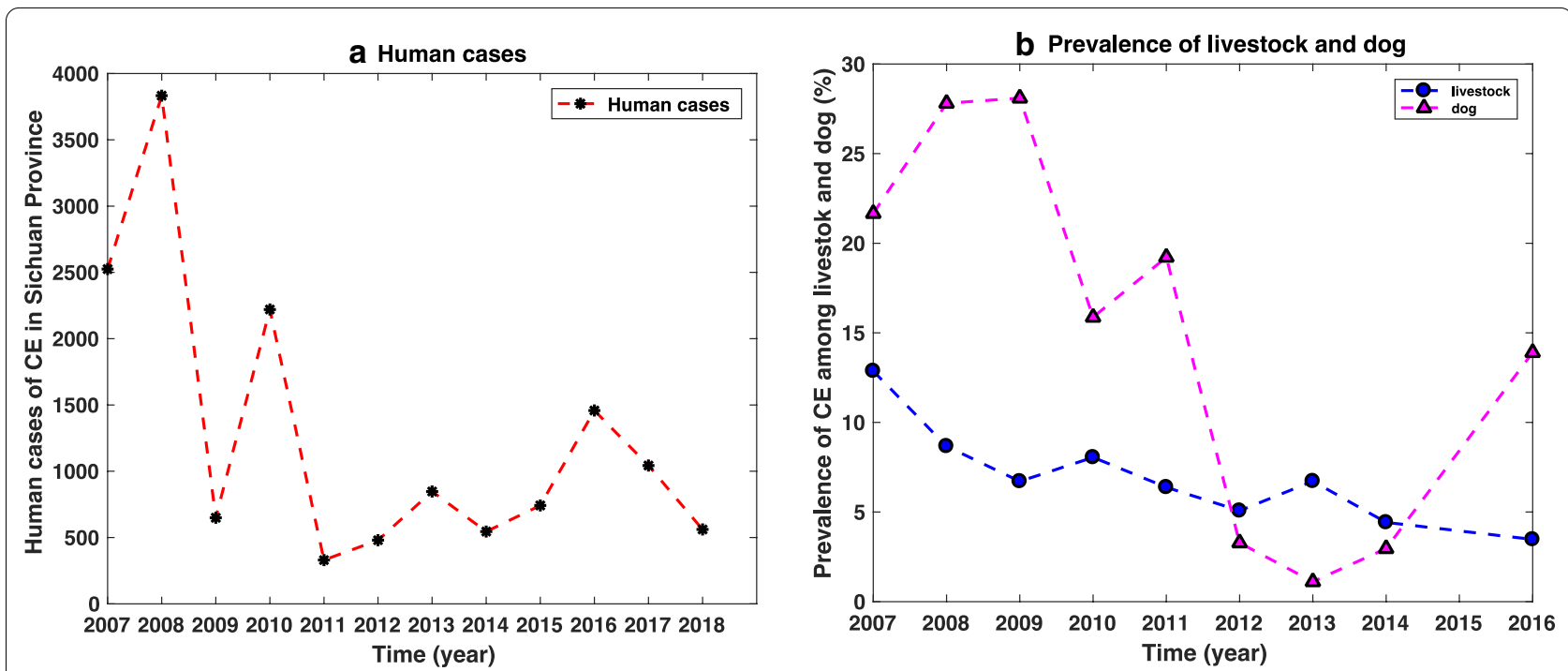

Fig. 1 Data of human CE cases, infection ratios of sheep and dogs in Sichuan Province, China from [11-13] 


\section{Methods}

Transmission model without optimal control

A compartmental model is developed by adding a compartment of the intermediate host (sheep) with vaccination immunity. Our model also considers other important control measures of CE, such as health education, deworming treatment for dogs, and disposals of stray dogs. The transmission cycle of E. granulosus occurs through the definitive and intermediate hosts, also involves the eggs of E. granulosus (Fig. 2).

As in [22], we still regard the domestic dog and stray dog as different definitive hosts. The total domestic dog population $\left(N_{d 1}(t)\right)$ and the total stray dog population $\left(N_{d 2}(t)\right)$ are divided into two groups: the susceptible population $\left(D_{1 s}, D_{2 s}\right)$ and the infectious population $\left(D_{1 i}, \mathrm{D}_{2 \mathrm{i}}\right)$, then $N_{d j}=D_{j s}(t)+D_{j i}(t)$ for $j=1,2$. Here, we assume that domestic dogs reproduce at a constant recruitment rate, based on the fact that the feed of domestic dogs is almost unchanged, while the excess puppies are mostly abandoned or given away [10]. Meanwhile, stray dogs are often under-fed and free to live [10], then we legitimately believe that stray dogs follow a Logistic growth due to limited food resources. Under these conditions, the growth of dogs can be expressed as following equations:

$$
\left\{\begin{array}{l}
\frac{d N_{d 1}}{d t}=A_{d 1}-d N_{d 1} \\
\frac{d N_{d 2}}{d t}=\left(b_{d 2}-d\right)\left(1-\frac{N_{d 2}}{K_{d 2}}\right) N_{d 2}-\theta N_{d 2}
\end{array}\right.
$$

where $A_{d 1}$ denotes the average annual recruitment rate of domestic dogs, d indicates the natural death rate of dogs, $b_{d 2}$ is the birth rate of stray dogs, $K_{d 2}$ reflects the food resources for stray dogs, and $\theta$ is the disposing rate of stray dogs. Since the stray dog keeps growing in natural setting, we assume that $b_{d 2}>(d+\theta)$.

As intermediate hosts, the sheep becomes infected by contacting with parasitic eggs. The current vaccination program carried out in western Sichuan Province is to implement mandatory immunization for all sheep. Generally, the vaccine injection procedure consists of two parts: (1) for newborn lambs, vaccinate these lambs once at birth, and then vaccinate them again one month later; (2) for adult sheep (greater than one year of age), vaccinate them once a year [23]. Meanwhile, the vaccination program with the Eg95 vaccine has proven to be an effective intervention against the disease [24]. Correspondingly, we divide the sheep population into susceptible individuals $L_{s}(t)$, infectious individuals $L_{i}(t)$, and vaccinated individuals $V(t)$. The density of E. granulosus eggs depends mainly on the number of infectious domestic and stray dogs, and also relates to the mortality rate of eggs in the environment. We consider $E(t)$ as the density of E. granulosus eggs, which comes only from the dumping of infectious domestic and stray dogs.

Based on the flow diagram in Fig. 2 and considering human as an incidental intermediate host, we formulate the following livestock-dog-egg life-cycle model:

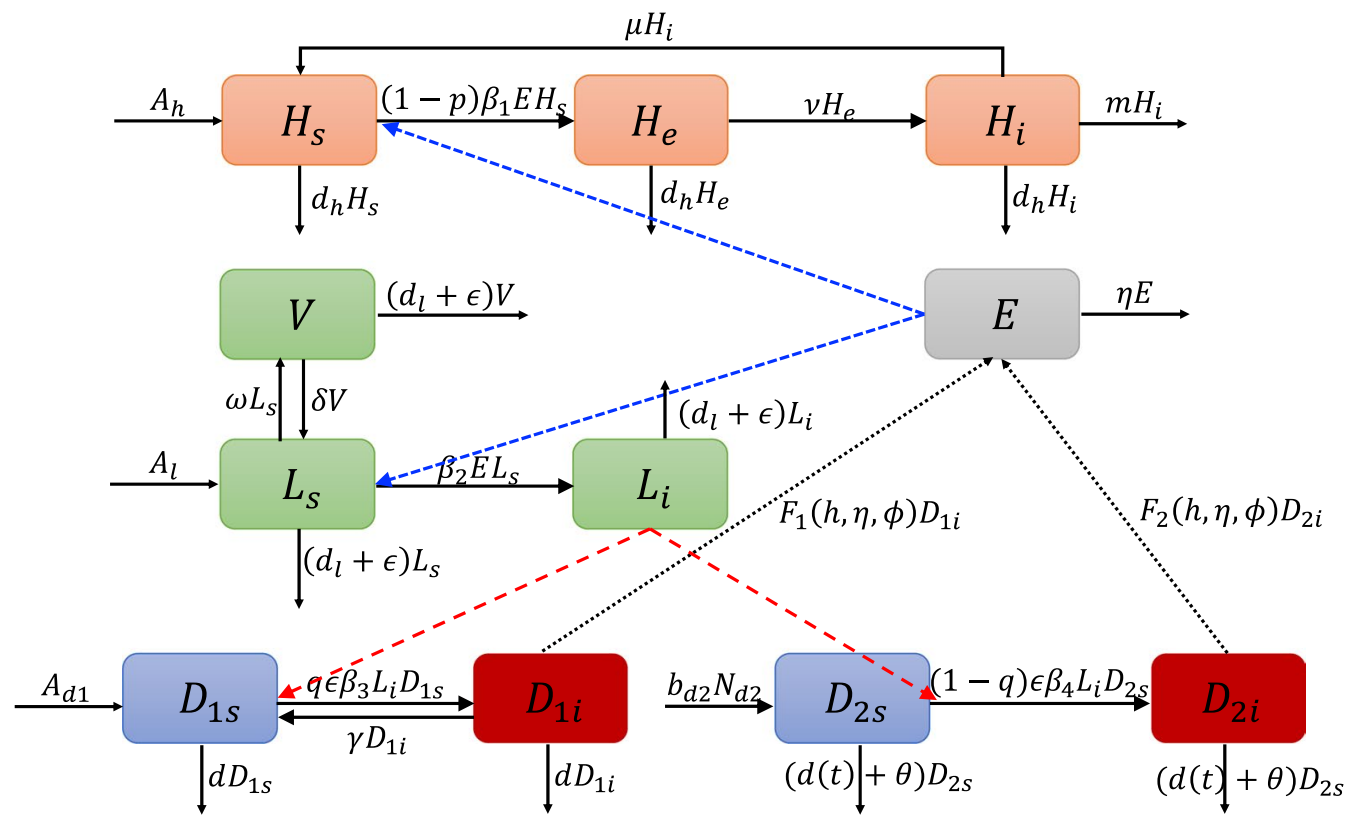

Fig. 2 Flux diagram of the Echinococcus granulosus in human, sheep and dogs 


$$
\left\{\begin{array}{l}
\frac{d L_{s}}{d t}=A_{l}-\beta_{2} E L_{s}-\omega L_{s}+\delta V-\left(\epsilon+d_{l}\right) L_{s} \\
\frac{d V}{d t}=\omega L_{s}-\delta V-\left(\epsilon+d_{l}\right) V \\
\frac{d L_{i}}{d t}=\beta_{2} E L_{s}-\left(\epsilon+d_{l}\right) L_{i} \\
\frac{d D_{1 s}}{d t}=A_{d 1}-q \epsilon \beta_{3} L_{i} D_{1 s}+\gamma D_{1 i}-d D_{1 s} \\
\frac{d D_{1 i}}{d t}=q \epsilon \beta_{3} L_{i} D_{1 s}-\gamma D_{1 i}-d D_{1 i} \\
\frac{d D_{2 s}}{d t}=b_{d 2} N_{d 2}-(1-q) \epsilon \beta_{4} L_{i} D_{2 s}-\theta D_{2 s}-\frac{\left(b_{d 2}-d\right) N_{d 2}}{K_{d 2}} D_{2 s}-d D_{2 s} \\
\frac{d D_{2 i}}{d t}=(1-q) \epsilon \beta_{4} L_{i} D_{2 s}-\theta D_{2 i}-\frac{\left(b_{d 2}-d\right) N_{d 2}}{K_{d 2}} D_{2 i}-d D_{2 i} \\
\frac{d E}{d t}=F_{1}(h, \eta, \phi) D_{1 i}+F_{2}(h, \eta, \phi) D_{2 i}-\eta_{1} E
\end{array}\right.
$$

In model (2), for the sheep populations, we use $A_{l}$ to represent the average annual recruitment rate, $\epsilon$ is the fraction of annual slaughtered sheep, $\omega$ is the vaccination rate, $\delta$ means the invalid sheep vaccination rate $(1 / \delta)$ represents the effective-immunity duration of the vaccine), $d_{l}$ denotes the natural death rate of sheep, $\beta_{2} E L_{s}$ describes the transmission of CE to sheep by the ingestion of $E$. granulosus eggs in the environment.

For the dog populations, $q$ is the livers intake fraction of domestic dogs, and $\gamma$ is the deworming recovery rate of infectious domestic dogs. In fact, sheep and cattle are still slaughtered in the traditional way and the abandoned livers are easily accessible to scavenging dogs, so the dog's infection rate also depends on the slaughter proportion $\epsilon$. We then separate $q \epsilon \beta_{3} L_{i} D_{1 s}$ and $(1-q) \epsilon \beta_{4} L_{i} D_{2 s}$ as the CE transmission of domestic dogs and stray dogs after ingesting cystcontaining organs of infectious livestock.

The E. granulosus eggs are produced in the worms of the dog and released with its feces. We follow the assumptions in [25] and use $F_{k}(h, \eta, \varphi)$ for the released rate from infectious $\mathrm{d}$ og $\mathrm{s}, F_{k}(h, \eta, \varphi)=\varphi(h / \eta)(1-\exp \{-\eta t k\})(k=1,2)$, where $\varphi$ denotes the proportion of worms that release eggs, $h$ is the egg released rate of one worm unit time, $t_{k}$ describes the average lifespan of domestic and stray dogs, $\eta$ is the parasite eggs mortality rate in the dog's small intestines, while $\eta_{1}$ represents the natural mortality rate of eggs in the environment. It is necessary to point out that, sheep vaccination and dog deworming are discrete intervention strategies, in this study, all intervention are assumed to be continuous deployment since the time scale here is selected as 'year'.

Furthermore, in order to raise public awareness, some moderate measures have been taken to protect against $C E$ such as human health education. We assume that human infection is linearly dependent on the amount of released eggs, and then we use $(1-p) \beta_{1} E H_{s}$ as the $\mathrm{CE}$ infection rate. When humans are infected by eggs, humans may take months or even years to show symptoms [26], we introduce the incubation period of infected (exposed) individuals, represented by $1 / \nu$. When a cyst in the human body is excised, the infectious individual recovers from the infection as recovery rate $\mu$. The total human population is separated into the classes of susceptible $H_{s}(t)$, exposed $H_{e}(t)$, and infectious $H_{i}(t)$. We have the following equations for human

$$
\left\{\begin{array}{l}
\frac{d H_{s}}{d t}=A_{h}-(1-p) \beta_{1} E H_{s}+\mu H_{i}-d_{h} H_{s} \\
\frac{d H_{e}}{d t}=(1-p) \beta_{1} E H_{s}-v H_{e}-d_{h} H_{e} \\
\frac{d H_{i}}{d t}=v H_{e}-\left(\mu+d_{h}+m\right) H_{i}
\end{array}\right.
$$

where $A_{h}$ is the constant recruitment rate of human population, $p$ denotes the influence coefficient of publicity measures, $\mathrm{m}$ and $d_{h}$ are the disease induced and natural death rate, respectively. Here all parameters are positive and their biological significance, default values, and reference resource are summarized in Table 1. Systems (2) and (3) are coupled, we will treat them together for the purpose of control and prevention of CE.

\section{Transmission model with optimal control}

The CE asserts a heavy burden to human health and the socio-economics, while the government has limited financial support for control and prevention against CE. So, endemic-level optimal control measures must be carefully assessed. To determine the optimal control strategy, we reformulate our model (2) and (3) to include time-dependent anti-CE control measures.

Since the CE cases of human and infectious rate of dogs are still fluctuating even under the implementation of existing time-independent control measures (see Fig. 1), it is more reasonable to develop the optimal control strategy involving time-dependent control measures. Then the publicity measure $p$ is set to be time-dependent $p(t)$ to describe the time-varying health education strategy. One other important step is the sheep vaccination program, we will use $\omega(t)$ to measure the reduction rate due to the sheep vaccine 
Table 1 Parameters of model (0.2) and (0.3) with default values

\begin{tabular}{|c|c|c|c|}
\hline Parameters & Biological definition & Value (range) & Source \\
\hline$A_{h}$ & Annual recruitment rate of human population & $7.482 \times 10^{4}$ & [35] \\
\hline$p$ & Influence coefficient of publicity measures & $0.1(0-1)$ & Set \\
\hline$\mu$ & Recovery rate of humans who are received surgery & 0.75 & [13] \\
\hline$d_{h}$ & Natural death rate of humans & $1 / 76.5$ & [35] \\
\hline$m$ & The mortality rate induced by $C E$ & 0.022 & [36] \\
\hline$\beta_{1}$ & Prevailing infection pressure between parasite eggs and humans & $1.004 \times 10^{-12}$ & Esti \\
\hline$v$ & Transition rate from exposed humans to infectious humans & $1 / 14$ & [13] \\
\hline$A_{l}$ & Annual recruitment rate of sheep & $6.88 \times 10^{5}$ & [35] \\
\hline$\beta_{2}$ & Prevailing infection pressure between parasite eggs and sheep & $1.08 \times 10^{-9}$ & Esti \\
\hline$\epsilon$ & Fraction of annual slaughtered sheep & $0.2(0.16-0.667)$ & Assumption \\
\hline$\omega$ & Vaccination rate of sheep & $0.4(0.2-1)$ & Set \\
\hline$\delta$ & Invalid sheep vaccination rate & $0.67(0.5-2)$ & Assumption \\
\hline$d_{1}$ & Natural death rate of sheep & 0.152 & [35] \\
\hline$A_{d 1}$ & Annual recruitment rate of domestic dogs & $2 \times 10^{5}$ & [35] \\
\hline$q$ & The proportion of domestic dogs intake the livers of sheep & $0.3(0-1)$ & Set \\
\hline$\beta_{3}$ & Prevailing infection pressure between infectious sheep and domestic dogs & $7.1 \times 10^{-9}$ & Esti \\
\hline$\gamma$ & Deworming recovery rate of infectious domestic dogs & $0.4(0.1-0.9)$ & Set \\
\hline$d$ & Natural death rate of dog & 0.08 & {$[37]$} \\
\hline$b_{d 2}$ & Birth rate of stray dog & 2 & Assumption \\
\hline$K_{d 2}$ & The food resources of stray dogs in the environment & $8.5 \times 10^{7}(6.5-10.5) \times 10^{7}$ & Assumption \\
\hline$\beta_{4}$ & Prevailing infection pressure between infectious sheep and stray dogs & $5 \times 10^{-8}$ & Esti \\
\hline$\theta$ & Disposing rate of stray dogs & $0.4(0.1-0.9)$ & Set \\
\hline$\phi$ & Egg released rate of one worm unit time & 42 & [38] \\
\hline$h$ & Worm produced rate per dog per year & 560 & [14] \\
\hline$\eta$ & Parasite eggs mortality rate in the dogs body & $12 / 5$ & [14] \\
\hline$t_{1}\left(t_{2}\right)$ & The average lifespan of domestic dogs (stray dogs) & $6(4)$ & [14] \\
\hline$\eta_{1}$ & Parasite eggs natural mortality in the environment & 10.42 & {$[37]$} \\
\hline
\end{tabular}

protection. Furthermore, the recovery rate of infectious domestic dogs under the vermicide $\gamma(t)$ and the disposing rate of stray dogs $\theta(t)$ represent the control efforts on domestic dogs and stray dogs, respectively.

Then, we modify model (2) and (3) as the following

$$
\left\{\begin{array}{l}
\frac{d H_{s}}{d t}=A_{h}-(1-p(t)) \beta_{1} E H_{s}+\mu H_{i}-d_{h} H_{s} \\
\frac{d H_{e}}{d t}=(1-p(t)) \beta_{1} E H_{s}-v H_{e}-d_{h} H_{e} \\
\frac{d H_{i}}{d t}=v H_{e}-\left(\mu+d_{h}+m\right) H_{i} \\
\frac{d L_{s}}{d t}=A_{l}-\beta_{2} E L_{s}-\omega(t) L_{s}+\delta V-\left(\epsilon+d_{l}\right) L_{s} \\
\frac{d V}{d t}=\omega(t) L_{s}-\delta V-\left(\epsilon+d_{l}\right) V \\
\frac{d L_{i}}{d t}=\beta_{2} E L_{s}-\left(\epsilon+d_{l}\right) L_{i} \\
\frac{d D_{1 s}}{d t}=A_{d 1}-q \epsilon \beta_{3} L_{i} D_{1 s}+\gamma(t) D_{1 i}-d D_{1 s} \\
\frac{d D_{1 i}}{d t}=q \epsilon \beta_{3} L_{i} D_{1 s}-\gamma(t) D_{1 i}-d D_{1 i}, \\
\frac{d D_{2 s}}{d t}=b_{d 2} N_{d 2}-(1-q) \epsilon \beta_{4} L_{i} D_{2 s}-\theta(t) D_{2 s}-\frac{\left(b_{d 2}-d\right) N_{d 2}}{K_{d 2}} D_{2 s}-d D_{2 s} \\
\frac{d D_{2 i}}{d t}=(1-q) \epsilon \beta_{4} L_{i} D_{2 s}-\theta(t) D_{2 i}-\frac{\left(b_{d 2}-d\right) N_{d 2}}{K_{d 2}} D_{2 i}-d D_{2 i} \\
\frac{d E}{d t}=F_{1}(h, \eta, \phi) D_{1 i}+F_{2}(h, \eta, \phi) D_{2 i}-\eta_{1} E
\end{array}\right.
$$

Here, our goal is to identify an integrated control strategy that jointly minimizes the number of infectious human as well as sheep population and the cost of control programs. Mathematically, using the system (4), we 
have developed an optimal control problem with the objective functional defined as

$$
\begin{aligned}
J(p(t), \omega(t), \gamma(t), \theta(t))= & \int_{0}^{T}\left(B_{0} H_{i}(t)+B_{1} L_{i}(t)+C_{1} p^{2}(t)\right. \\
& \left.+C_{2} \omega^{2}(t)+C_{3} \gamma^{2}(t)+C_{4} \theta^{2}(t)\right) d t
\end{aligned}
$$

where $B_{0}$ and $B_{1}$ represent, respectively, the weight constants of the infectious human and infected sheep populations. $C_{1}, C_{2}, C_{3}, C_{4}$ are balancing coefficients transforming the integral into cost expended over a finite time period of $\mathrm{T}$ years. The terms $C_{1} p^{2}(t)$ and $C_{2} \omega^{2}(t)$ describe the total costs associated with human population's health education and vaccination of whole sheep population, respectively. $C_{3} \gamma^{2}(t)$ and $C_{4} \theta^{2}(t)$ read the costs for anthelmintic control of domestic dogs and disposing of stray dogs. Here the objective functional $J(p(t), \omega(t), \gamma(t), \theta(t))$ measures the total economic loss caused by CE. That is, we need to determine an optimal control $\left(p^{*}(t), \omega^{*}(t), \gamma^{*}(t), \theta^{*}(t)\right)$ such that

$$
J\left(\left\{p^{*}(t), \omega^{*}(t), \gamma^{*}(t), \theta^{*}(t)\right\}\right)=\min _{U}(\{p(t), \omega(t), \gamma(t), \theta(t)\}),
$$

where $U=\left\{(p(t), \omega(t), \gamma(t), \theta(t)) \mid\right.$ s.t. $0 \leq p(t) \leq p_{\max }, 0$ $\left.\leq \omega(t) \leq \omega_{\max }, 0 \leq \gamma(t) \leq \gamma_{\max }, 0 \leq \theta(t) \leq \theta_{\max }, t \in[0, T]\right\}$ is the feasible decision space or the control set, which is closed and convex.

Note that the integrand of the objective functional given by (5) is convex on $U$, the model is linear in the control variables and is bounded by a linear system in the state variables. By Theorem 4.1 and Corollary 4.1 in [27], there exists an optimal control $\left(p^{*}(t), \omega^{*}(t), \gamma^{*}(t), \theta^{*}(t)\right)$, such that (6) holds, and the state solution corresponding to the optimal control reads $H s^{*}(t), H e^{*}(t), H i^{*}(t), L_{s}^{*}(t), V^{*}(t), L_{i}^{*}(t), D_{1 s}^{*}(t), D_{1 i}^{*}(t)$, $D_{2 s}^{*}(t), D_{2 i}^{*}(t), E^{*}(t)$, find more details in the Additional file 1.

\section{Parameter values setting}

The values of parameters involved in the models (2) and (3) were obtained from references and estimation, see Table 1. For simulations of the model (4), based on the official survey reports and personal communication with professionals, we took the weights $B_{2}=15,000$ and $B_{1}=300$, which means that more effort is given to the minimization of the infectious humans than that to the reduction of infected sheep. We set $p=0.1, \omega=0.3, \gamma=0.65$ and $\theta=0.43$ due to the current control status in western Sichuan Province. Meanwhile, from the survey report [28], the cost for health education in Sichuan was about 300,000 RMB per year and the cost for sheep vaccination and the treatment of infectious disease was about 896,000 RMB per year. We then assumed that the total cost for each control measure is same, i.e., $C_{1}=C_{2}=C_{3}=C_{4}=300,000$. Since the controls would not be $100 \%$ effective, without any loss of generality, we assumed that the upper bounds of the four control measures are set to be 0.7, i.e., $p_{\max }=\omega_{\max }=\gamma_{\max }=\theta_{\max }=0.7$.

\section{Sensitivity analyses and evaluation of optimal control strategy}

Sensitivity analyses were conducted by evaluating the partial rank correlation coefficients (i.e., PRCCs) for various parameters against the basic reproduction number $R_{0}$ over time. The parameters considered here were control measure $(\omega, \gamma, \theta)$, annual sheep slaughtered fraction $(\varepsilon)$, invalid sheep vaccination rate $(\delta)$, and food resources of stray dogs $\left(K_{d 2}\right)$. Contour plot for $R_{0}$ was used to evaluate the effect of $\delta$ and $K_{d 2}$ on disease prevalence and control outcomes. Furthermore, by using "forward-backward sweep method" [29], we numerically simulated the implementation of optimal control strategy for significant reduction of infections of human $\left(H_{i}\right)$, sheep $\left(L_{i}\right)$, domestic $\operatorname{dog}\left(D_{1 i}\right)$, and stray $\operatorname{dog}\left(D_{2 i}\right)$.

\section{Results}

\section{Theoretical results}

Obviously, the system always has a trivial equilibrium $E_{d f 0}$ and a disease-free equilibrium (DFE) $E_{d f e}$, which reads

$$
\begin{aligned}
& E_{d f 0}=\left(H_{s}^{0}, 0,0, \mathrm{~L}_{\mathrm{s}}^{0}, \mathrm{~V}^{0}, 0, \mathrm{D}_{1 \mathrm{~s}}^{0}, 0,0,0,0\right), \\
& E_{d f e}=\left(H_{s}^{0}, 0,0, \mathrm{~L}_{\mathrm{s}}^{0}, \mathrm{~V}^{0}, 0, \mathrm{D}_{1 \mathrm{~s}}^{0}, 0, \mathrm{D}_{2 \mathrm{~s}}^{0}, 0,0\right),
\end{aligned}
$$
$\begin{array}{rr}\text { where } H_{s}^{0}=A_{h} / d_{h}, & L_{s}^{0}=\alpha A_{1} /\left(d_{l}+\epsilon\right), \\ V^{0}=(1-\alpha) A_{l} /\left(d_{l}+\epsilon\right), & D_{1 s}^{0}=A_{d 1} / d, \\ D_{2 s}^{0}=\left(b_{d 2}-d-\theta\right) K_{d 2} /\left(b_{d 2}-d\right), & \text { and }\end{array}$ $\alpha=\left(\delta+\epsilon+d_{l}\right) /\left(\omega+\delta+\epsilon+d_{l}\right)$.

Following the next generation matrix method developed by [30], the new infection terms and the remaining transfer terms are given respectively by $F$ and $V$, and the basic reproduction number $R_{0}$, which is calculated from $\rho\left(F V^{-1}\right)$ (see the Additional file 1 ), is given by

$$
\sqrt[3]{\frac{\beta_{2} \bar{\beta}_{3} \alpha A_{l} A_{d 1} F_{1}}{\left(\epsilon+d_{l}\right)^{2}(\gamma+d) d \eta_{1}}+\frac{\beta_{2} \bar{\beta}_{4} \alpha A_{l}\left(b_{d 2}-d-\theta\right) K_{d 2} F_{2}}{\left(\epsilon+d_{l}\right)^{2}\left(b_{d 2}-d\right) b_{d 2} \eta_{1}}=\sqrt[3]{R_{10}^{3}+R_{20}^{3}}}
$$


where $\quad \bar{\beta}_{3}=q \epsilon \beta_{3}, \quad \bar{\beta}_{4}=(1-q) \epsilon \beta_{4}$,

$F_{k}=F_{k}(h, \eta, \phi), k=1,2$ and

$$
\begin{aligned}
& R_{10}=\sqrt[3]{\underbrace{F_{1} / \eta_{1}}_{\text {eggs by per domestic dog }} \cdot \underbrace{\left(\beta_{2} \alpha A_{l}\right) /\left[(\gamma+d)\left(\epsilon+d_{l}\right)\right]}_{\text {infected sheep by eggs }} \cdot \underbrace{\left(\bar{\beta}_{3} A_{d 1}\right) /\left[\left(\epsilon+d_{l}\right) d\right]}_{\text {infected domestic dogs }}} \\
& R_{20}=\sqrt[3]{\underbrace{F_{2} / \eta_{1}}_{\text {eggs by per stray dog }} \cdot \underbrace{\left(\beta_{2} \alpha A_{l}\right) /\left[b_{d 2}\left(\epsilon+d_{l}\right)\right]}_{\text {infected sheep by eggs }} \cdot \underbrace{\left(\bar{\beta}_{4} K_{d 2}\left(b_{d 2}-d-\theta\right) /\left[\left(\epsilon+d_{l}\right)\left(b_{d 2}-d\right)\right]\right.}_{\text {infected stray dogs }}}
\end{aligned}
$$

The basic reproduction number $R_{0}$ is a key indicator measuring the average new infections produced by infectious dogs as presented in [31]. $R_{10}$ is the average number of secondary infectious individuals (domestic dogs) only generated by an infectious domestic dogs [30]. Similarly, $R_{20}$ is the average number of the infectious individuals (stray dogs) which infected only by stray dogs. The cube root measures the infection power of $\mathrm{CE}$ into three-step transmission cycle, from infectious dogs to E. granulosus eggs, to sheep, and then to infectious dogs.

We obtained the existence of the domestic dog-drive endemic equilibrium and the endemic equilibrium through solving the systems (2) and (3) for $R_{10}>1$ and $R_{0}>1$, respectively. In addition, we theoretically proved that the trivial and domestic dog-drive endemic equilibria are always unstable, the disease-free equilibrium is locally asymptotically stable if $R_{0}<1$ and further it is globally asymptotically stable when $R_{0}<1$. Furthermore, the global stability of the endemic equilibrium proved by the methods in $[32,33,39-41]$. The results are established in the Additional file 1.

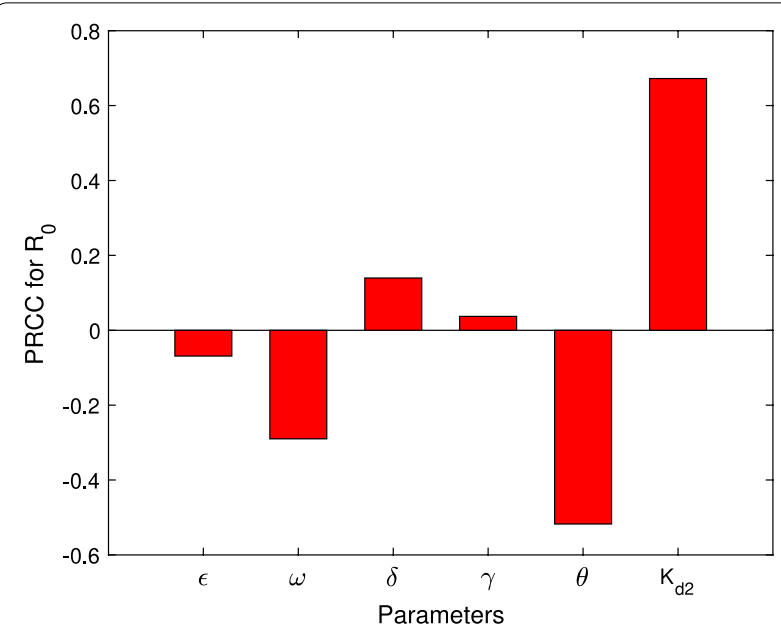

Fig. 3 Sensitive analysis of the basic reproduction number $R_{0}$ via parameters

\section{Simulation results \\ Sensitive analysis and evaluation of key parameters}

The result illustrated in Fig. 3 suggests that, for the chosen 'control parameter' ranges, $R_{0}$ is more sensitive to $\theta$ and $\omega$, followed by $\gamma$. In particular, it should be noted that $K_{d 2}$ and $\delta$ show a significant impact on $R_{0}$ (the values of the PRCCs for $K_{d 2}$ and $\delta$ are big), while these two factors are always neglected. In addition, these results indicate that both the administration and living resources of stray dogs contribute more to the transmission of $\mathrm{CE}$. The analyses are demonstrated in Fig. 4.

The contour plots of $R_{0}$ responding to the invalid sheep vaccination rate $(\delta)$ and the vaccination rate $(\omega)$ for different stray dog resources $\left(K_{d 2}\right)$ are showed in Fig. $4 \mathrm{a}$, b. It indicates that, when the stray dog's food become richer, in order to guarantee $R_{0}<1$, we must decrease the invalid sheep vaccination rate and increase the vaccination rate. For example, when $K_{d 2}=8 \times 10^{7}$, the minimum of $\omega$ such that $R_{0}<1$ is 0.38 , while when $\omega \geq 0.8, R_{0}<1$ for any $\delta$ even if $\delta$ reaches the maximum value 1.5 (Fig. 4a; when $K_{d 2}=9.5 \times 10^{7}$, in order to have $R_{0}<1$ the minimum of $\omega$ now is 0.6 and $\delta$ must be less than 1.1 (see the dashed line in Fig. 4b). In addition, Fig. 4a, b show that, in order to achieve the same effectiveness of vaccination, it is more difficult to reduce $R 0$ from 1.1 to 1 when $K_{d 2}=9.5 \times 10^{7}$ (e.g., for $\delta=0.5$ in (a)-(b), the solid line in Fig. $4 \mathrm{~b}$ is longer).

The joint effects of $\gamma$ and $\theta$ on decreasing $R_{0}$ for $K_{d 2}=8 \times 10^{7}$ or $9.5 \times 10^{7}, \delta=1$ or 0.67 are illustrated in Fig. 4c-f, respectively. We observe that $R_{0}$ is more sensitive to $\theta$ than $\gamma$ in all four cases. When $K_{d 2}$ or $\delta$ is bigger, in order to reduce $R_{0}$ such that $R_{0}<1$, one has to carry out intensive control of dogs (especially stray dogs). It is also observed that, for more food resources of stray dogs and higher invalid sheep vaccination rate, it is much more difficult to reduce $R_{0}$ such that $R_{0}<1$ (e.g., in Fig. $4 \mathrm{~d}, R_{0}<1$ only when $\theta>0.6$ ). In general, more food resources of stray dog and higher invalid sheep vaccination rate may have more negative effect on the $C E$ control. 

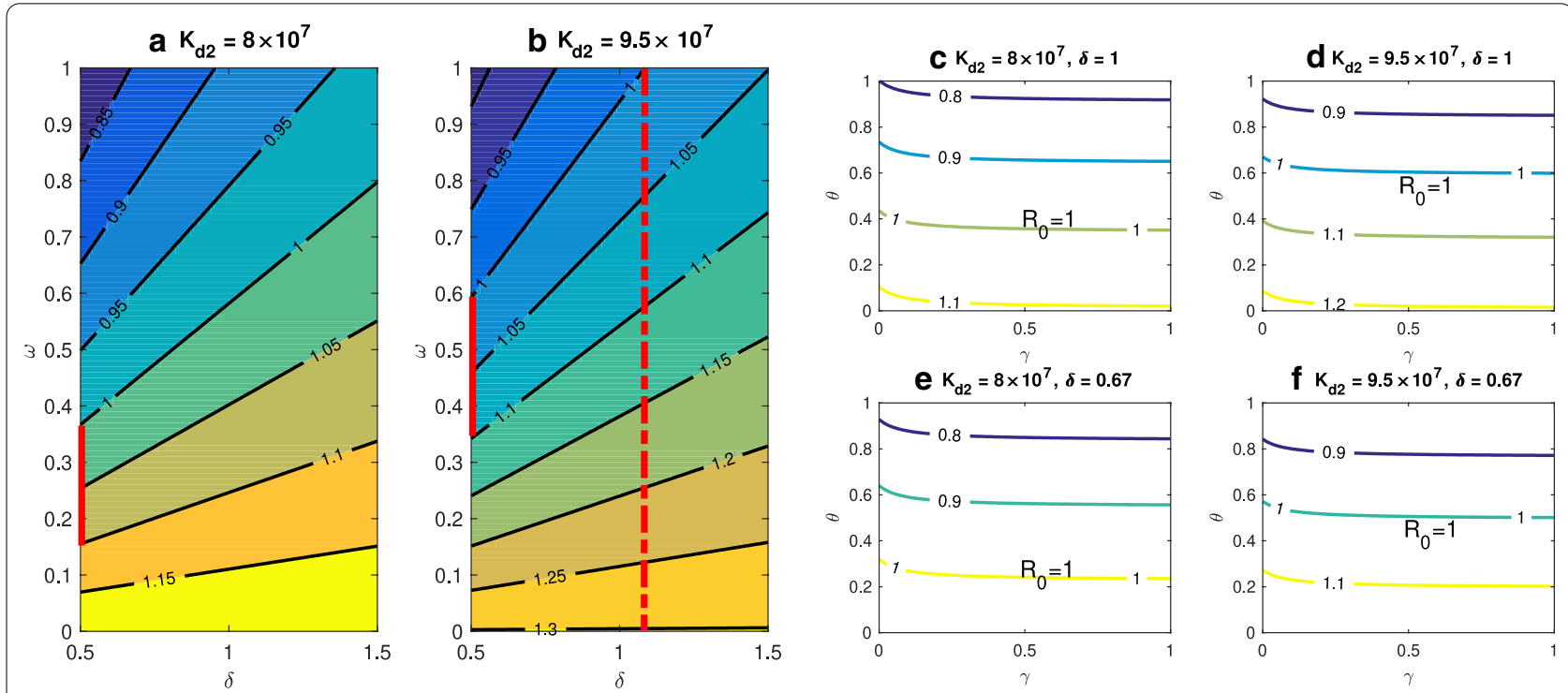

Fig. 4 Contour plots of $R_{0}$. $\mathbf{a}, \mathbf{b}$ the $(\delta, \omega)$ planes with various stray dogs'food resources $K_{d 2}$. The contour plots measure the effectiveness of invalid sheep vaccination rate $(\delta)$ and vaccination rate $(\omega)$ on reducing $R_{0}$. $\mathbf{c}-\mathbf{f}$ the $(\gamma, \theta)$ planes respond to different $K_{d 2}$ as well as $\delta$. The contour plots depict the effectiveness of domestic dog's recovery rate $(\gamma)$ and stray dog's disposing rate $(\theta)$ on decreasing $R_{0}$. Other parameters are listed in the Table 1

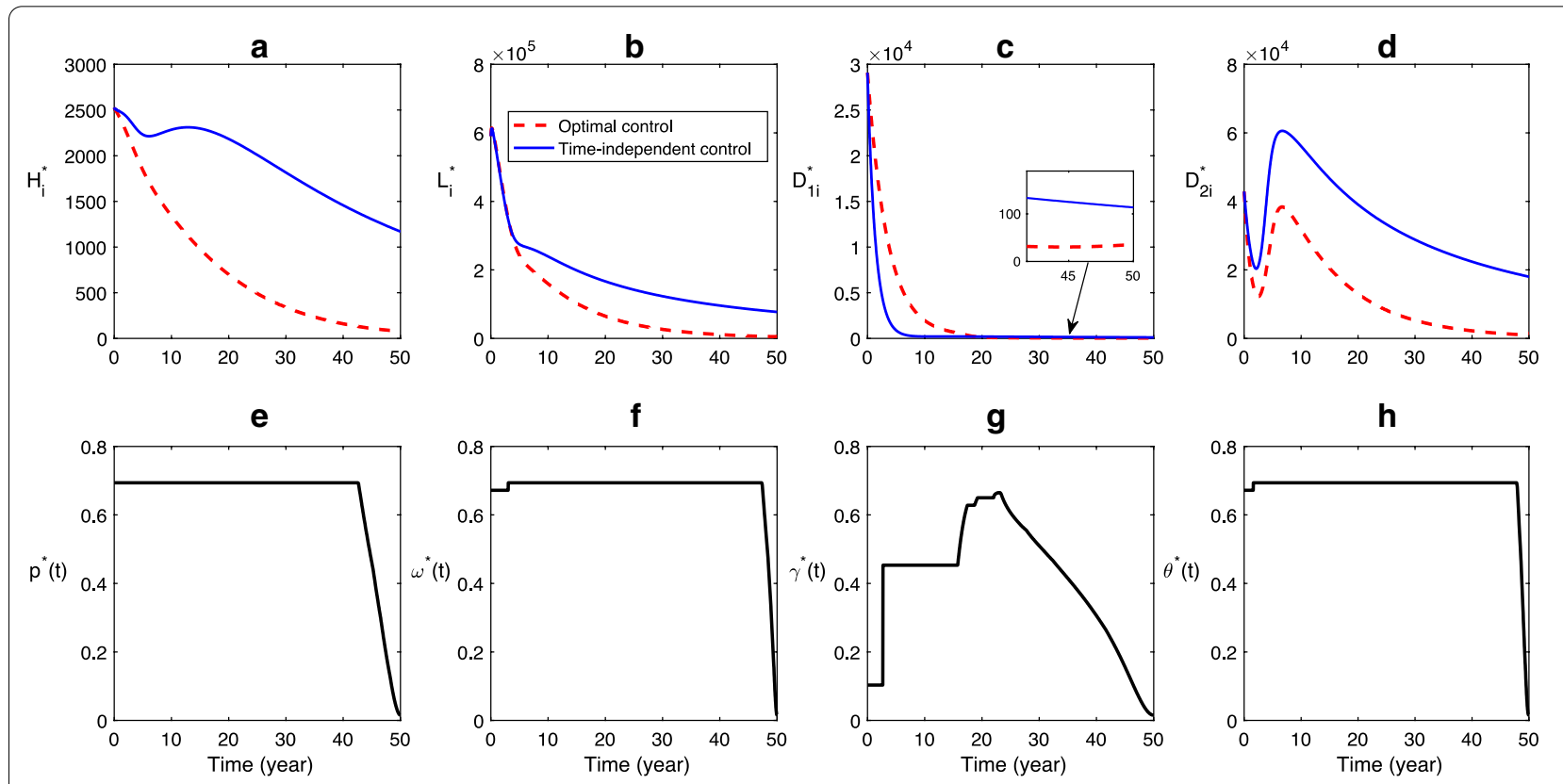

Fig. 5 Advantage of optimal control. a-d Time series of the infections corresponding to the time-independent control (solid curves) and the optimal control (dashed curves). $\mathbf{e}-\mathbf{h}$ The optimal control strategies for health education $\left(p^{*}(t)\right)$, sheep vaccination $\left(\omega^{*}(t)\right)$, domestic dog deworming $\left(\gamma^{*}(t)\right)$ stray dog disposing $\left(\theta^{*}(t)\right)$. Here $C_{1}=C_{2}=C_{3}=C_{4}=3 \times 10^{5}$, other parameter values are listed in Table 1, and the $H_{s}(0)=4580000, H_{e}(0)=26058, H_{i}(0)=2525, L_{s}(0)=1958000, V(0)=2038000, L_{i}(0)=590321, D_{1 s}(0)=$ initial conditions are $96900, D_{1 i}(0)=29090, D_{2 s}(0)=145350, D_{2 i}(0)=42892, E(0)=271400000$. time-independent control, $p=0.1, \omega=0.3, \gamma=0.65$ and $\theta=0.43$. 


\section{Advantage of optimal control}

The time-series dynamical scenarios of infectious hosts are performed for the system with time-independent control (solid curves in Fig. 5(a-d)) and with the optimal control efforts (dashed curves in Fig. 5(ad)), respectively. Figure $5(\mathrm{e}-\mathrm{h})$ illustrates the optimal control strategy, which is obtained by the forwardbackward sweep Runge- Kutta method [28]. It is observed that, in Fig. 5(a,b,d), the dash curve is always below the solid curve, while, in Fig. 5(c), the dash curve is above the solid curve first and then falls below the solid curve. These facts imply that the time-independent controls cannot effectively control CE transmission, while the optimal control strategy is more effective than the time-independent control, which can significantly reduce the infections and can eliminate infections of all hosts eventually. In order to minimize both the CE infections and the economic losses, in the optimal control strategy, the health education, sheep vaccination, and stray dog disposal are supposed to be kept at the maximum level for most time and finally decrease sharply (Fig. 5e, f, h); due to the less amount of domestic dogs, the domestic dog deworming increases first to its maximum and then decreases Fig. $5 \mathrm{~g}$ rather than keeps at a constant high level in the time-independent control (i.e., $\gamma=0.65$ ). The number of infectious domestic dogs corresponding to the optimal control is still less than that corresponding to the time-independent control (Fig. 5c). It implies again that the optimal control strategy is more realistic and effective.

\section{Effect of control cost}

In the following numerical experiments, in order to further characterize the optimal control strategy by investigating the effect of different control costs $C_{i}(i=1,2,3,4)$ on the effectiveness of optimal control, we took $C_{i}=3 \times 10^{5}$ or $3 \times 10^{6}, i=1,2,3,4$, and leave other parameters $C_{j}=3 \times 10^{5}, j \neq i, j=1,2,3,4$.

Compared to the scenarios with lower cost $\left(C_{1}=3 \times 10^{5}\right)$, when the cost of health education is higher $\left(C_{1}=3 \times 10^{6}\right)$, the final size (number of infection at the end of control period, i.e., $t=50$ ) of human infection is slightly higher while the final sizes of both sheep and dog infections are lower (Fig. 6). The reason is that the control effort for human health education and domestic dog deworming decreases due to the higher cost of health education, while the sheep vaccination and stray dog disposal increase (Fig. 6e-h). Moreover, although the effort for domestic dog deworming decreases when $C_{1}=3 \times 10^{6}$, the final infection size of domestic dogs becomes lower (Fig. 6c).

Similarly, we investigate the effects of different costs of sheep vaccination, domestic dog deworming, and stray dog disposing on the CE control (Figs. 7,
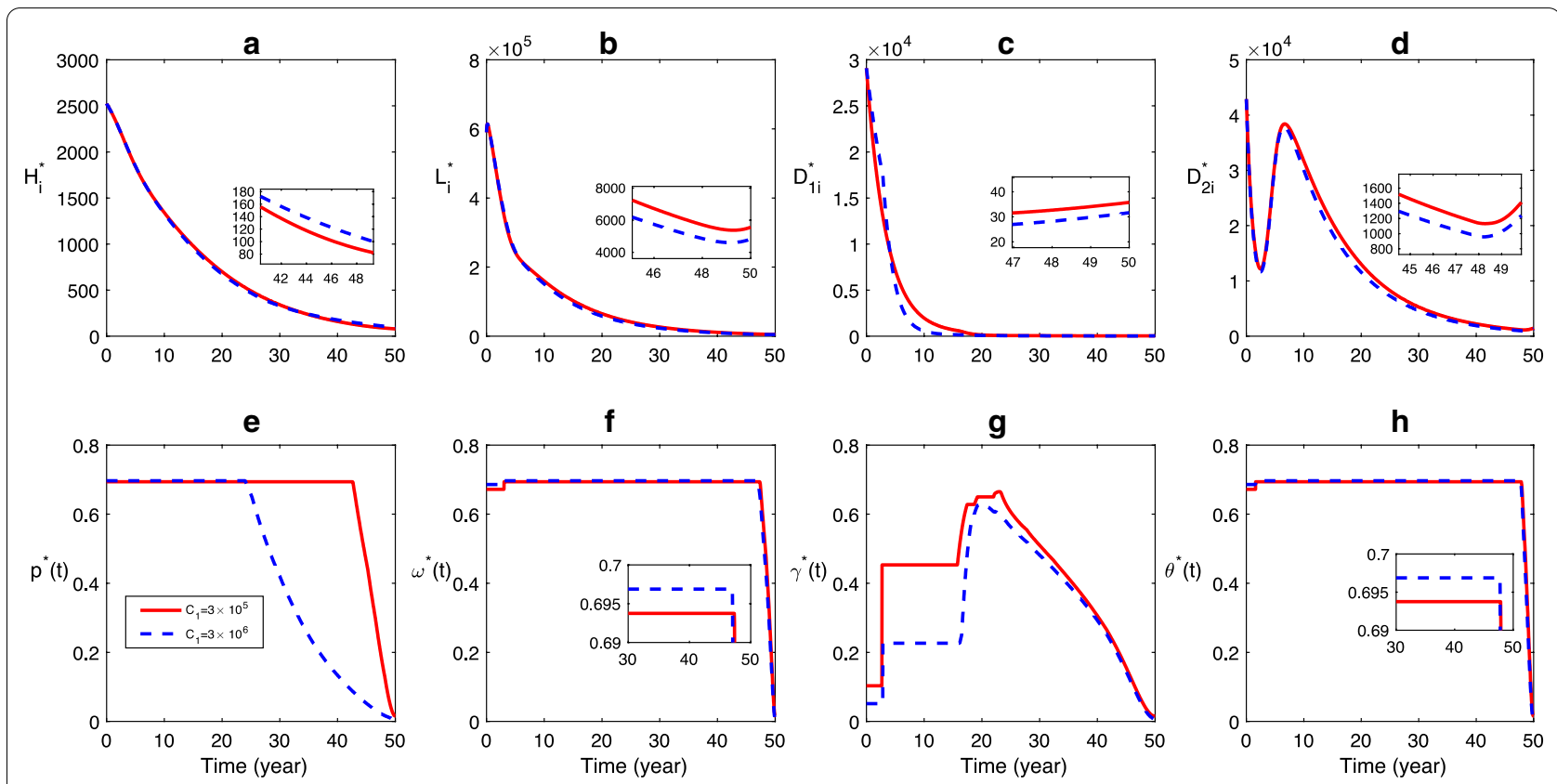

Fig. 6 Effect of control cost for health education: a-d Time series dynamics of infections, $\mathbf{e}-\mathbf{h}$ The optimal control strategy. The solid and dashed curves represent the scenarios for $C_{1}=3 \times 10^{5}$ and $C_{1}=3 \times 10^{6}$, respectively. Here $C_{2}=C_{3}=C_{4}=3 \times 10^{5}$, the values of other parameters are listed in Table 1, and the initial conditions are same with those in Fig. 5 

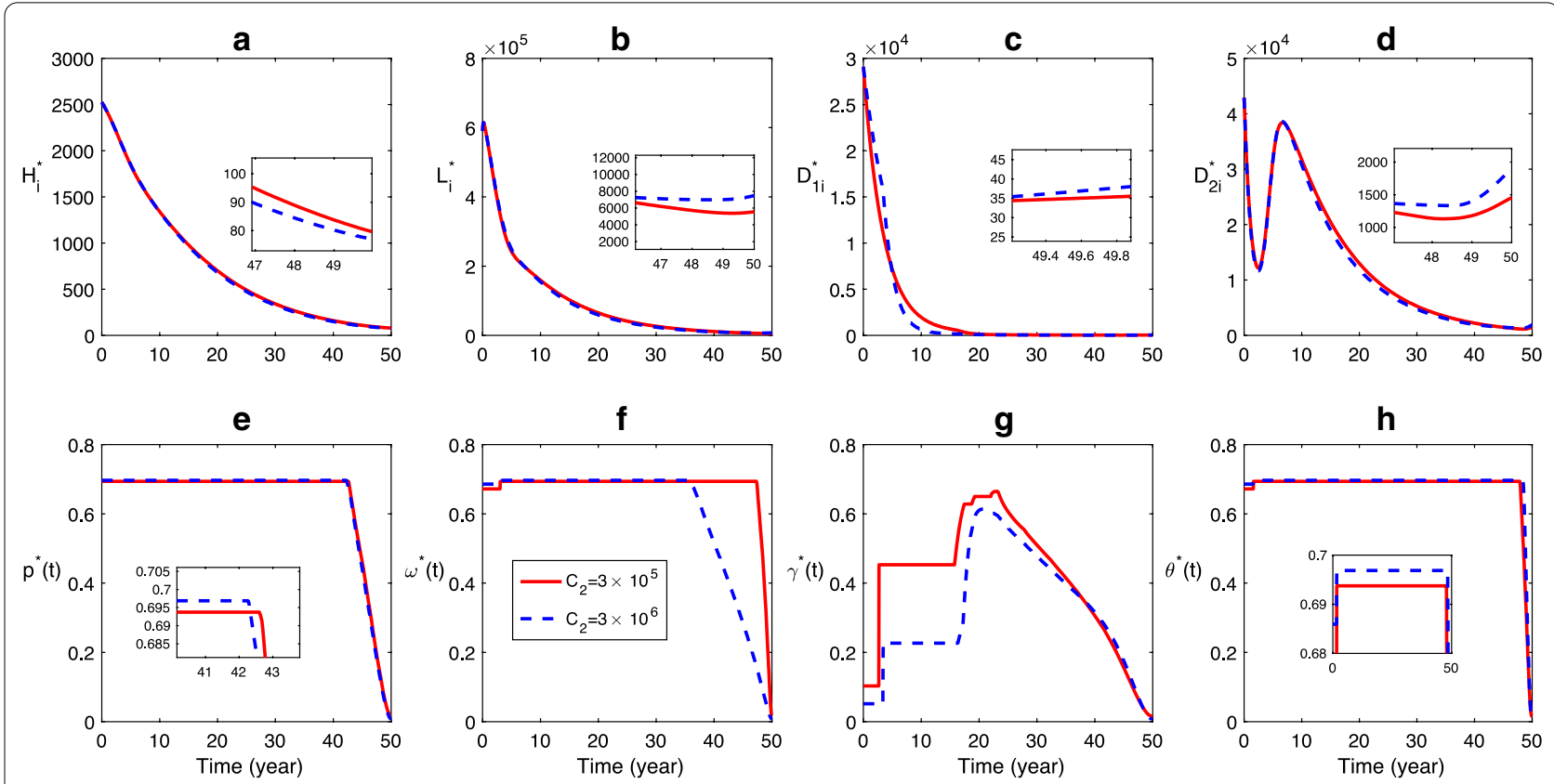

Fig. 7 Effect of control cost for sheep vaccination: $\mathbf{a}-\mathbf{d}$ Time series dynamics of infections, $\mathbf{e}-\mathbf{h}$ The optimal control strategy. The solid and dashed curves correspond to the scenarios for $C_{2}=3 \times 10^{5}$ and $C_{2}=3 \times 10^{6}$, respectively. Here $C_{1}=C_{3}=C_{4}=3 \times 10^{5}$, the values of other parameters and the initial conditions are same with those in Fig. 6

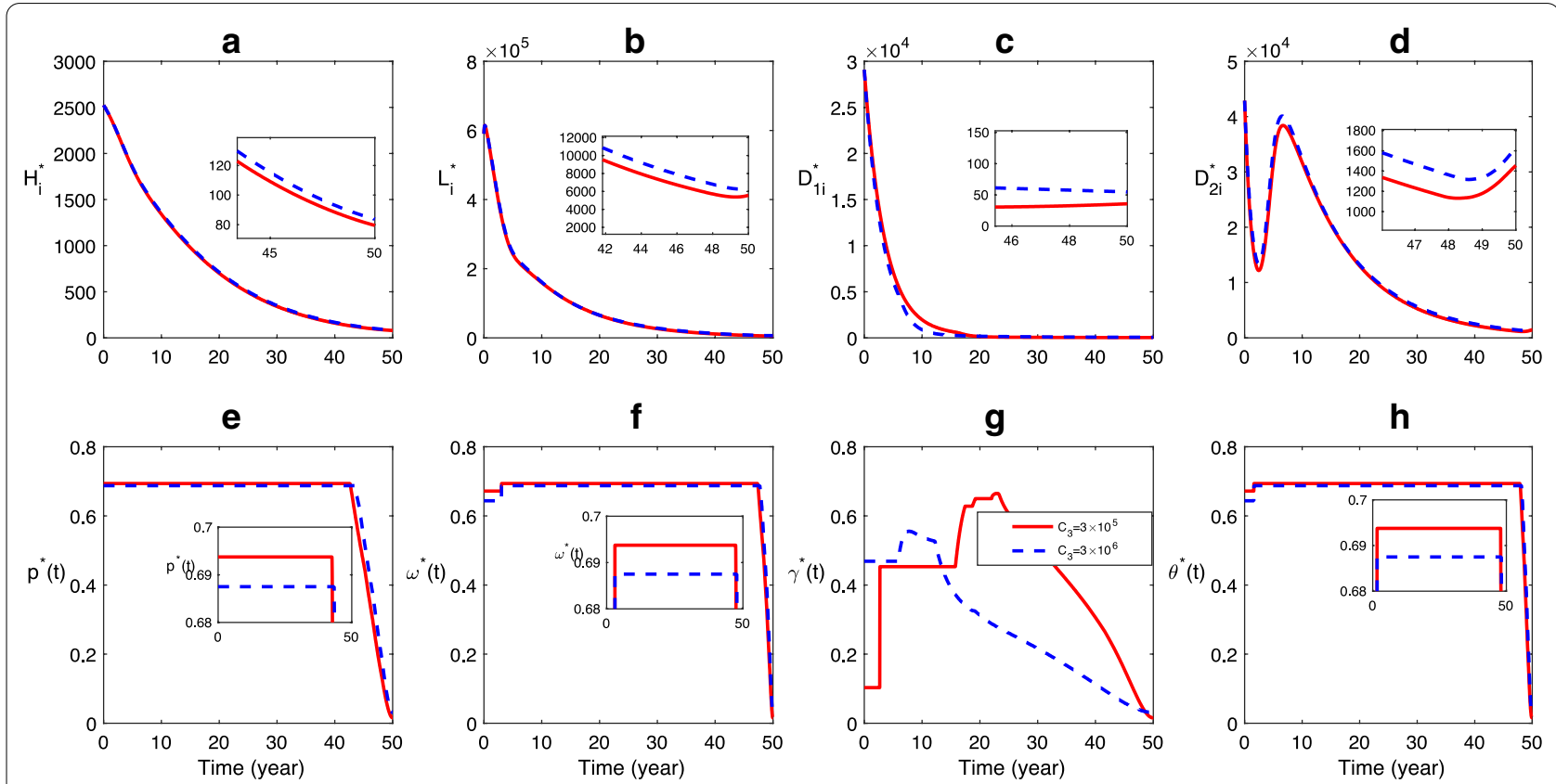

Fig. 8 Effect of control cost for domestic dog deworming: a-d Time series dynamics of infections, $\mathbf{e}-\mathbf{h}$ The optimal control strategy. The solid and dashed curves correspond to the scenarios for $C_{3}=3 \times 10^{5}$ and $C_{3}=3 \times 10^{6}$, respectively. Here $C_{1}=C_{2}=C_{4}=3 \times 10^{5}$, the values of other parameters and the initial conditions are same with those in Fig. 6

8, 9). Figure 7 shows that, higher cost of sheep vaccination leads to less control effort for sheep vaccination, domestic dog deworming, and stray dog disposing but more effort for long-term human health education (Fig. 7e-h). Due to the variation of those controls, the final size of human cases declines lightly while the final size of other infections do not increase much comparing to the case with lower cost 

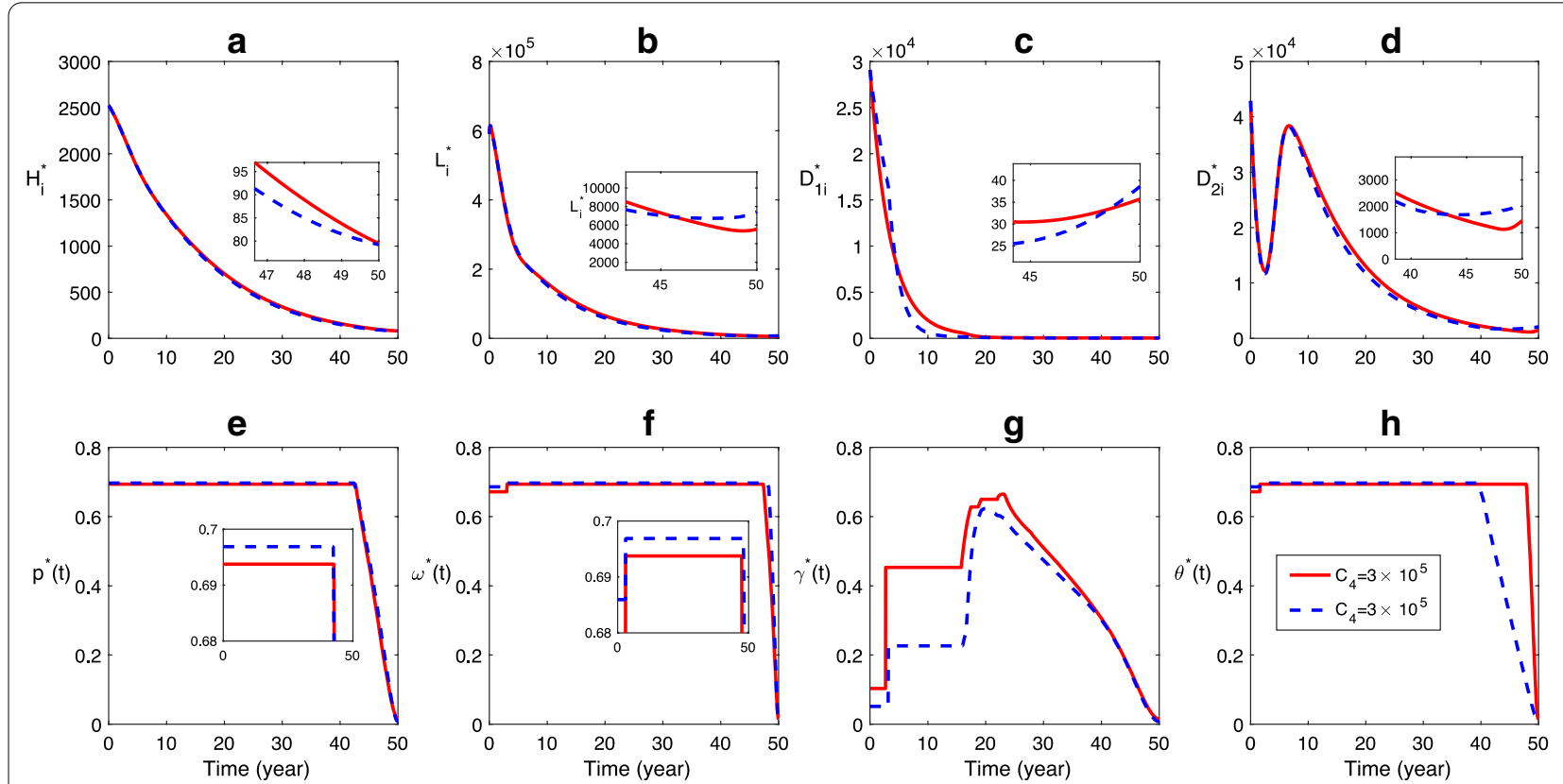

Fig. 9 Effect of control cost for stray dog disposing: a-d Time series dynamics of infections, $\mathbf{e}-\mathbf{h}$ The optimal control strategy. The solid and dashed curves correspond to the scenarios for $C_{4}=3 \times 10^{5}$ and $C_{4}=3 \times 10^{6}$, respectively. Here $c_{1}=C_{2}=C_{3}=3 \times 10^{5}$, the values of other parameters and the initial conditions are same with those in Fig. 6

of sheep vaccination (Fig. $7 \mathrm{a}-\mathrm{d}$ ). The increase of cost for domestic dog deworming reduces the effort for all the four control measures (Fig. 8e-h), which is followed by an increase of the final size in all infections (Fig. 8a-d). In addition, when the cost for stray dog control is higher, the final size of human cases decreases while the final sizes of other host infections become higher than those of the situation with lower cost of stray dog disposal (Fig. 9).

\section{Discussion}

The transmission of CE has been a growing public concern in China. Particularly, despite the effort of public health personals to strictly enforce the control measures proposed in 2006, the CE prevalent scenarios in western Sichuan Province for both human and dog exhibit an abnormal upward trend between 2007 and 2016 (see Fig. 1). Several possible factors that resulted in this unexpected trend include the effects of time-independent control, the effectiveness of sheep vaccination, and stray dog activities [1, 10, $24]$. Therefore, in addition to examining the transmission mechanism, we also explore effects of two key factors $\left(K_{d 2}\right.$ and $\left.\delta\right)$ on the CE transmission and the optimal control strategy. The model developed in this study extends the model in [22] by containing vaccinated sheep population and considering the crowding effect of stray dogs. The inclusion of vaccinated sheep immunity and stray dog's logistic-growth assumption provides a more realistic illustration of CE transmission in western Sichuan Province.

Both analytical and simulation results suggest the importance of the food resources of stray dogs and the invalid sheep vaccination rate in determining the control outcomes. As shown in 3 and Fig. 4, the abundance of stray dog's food resources restrains the elimination of $C E$ (i.e., make it more difficult to reduce $R_{0}$ less than 1 ), which is consistent with the result in [22]. The simulation results for optimal control show that the optimal controls are capable of reducing all infections to zero, and is more effective than the timeindependent controls (Fig. 5). Note that, to minimize both the infections and control costs, the control of sheep and stray dogs should maintain at a high level throughout the intervention, while the control efforts of domestic dogs can be reduced in the later stage to save resources.

Furthermore, the scenarios shown in Figs. 6, 7, 8, 9 indicate that, the different costs for these three control measures also contribute mildly to the reduction of all infections. The higher cost of human education could lead to a higher final size of human infections (see (a)-(d) in Fig. 6). It suggests that increasing the cost of health education could not help to control 
human $\mathrm{CE}$, but possibly reduce the final size of infection of sheep, domestic dog and stray dog. Hence, it is ineffective to reduce human infection by enhancing the financial effort for human health education. While the greater control cost of sheep and stray dog can make the final size of human infections be smaller, and the higher control cost of domestic dog deworming will result in a larger final size of all infections. Hence, sheep vaccination and stray dog disposal are more effective to control human CE infection, which is consistent with the results of sensitive analysis. Therefore, in order to control CE in all hosts, it is reasonable and acceptable to emphasize more on sheep vaccination and control of stray dogs, and then reduce the financial effort of the domestic dog deworming.

Although the numerical simulations show that $\mathrm{CE}$ may be effectively controlled by adopting the control strategies, the values of weight coefficients in the objective functional are usually subjectively determined. Note that, to apply the tools developed here, one would need to know estimates of actual costs and upper bounds on the controls. The roadmap of elimination programme of echinococcosis has been defined by Chinese government. Currently, there is a 10 year timeframe to reach the goal of "Healthy China 2030" [34]. The elimination of Echinococcosis by 2030 is an urgent task. In most portfolios studies, cost is one of the major factors to determine the successful strategy. Comprehensive consideration including the political may has higher impact than financing cost, then, sufficient resources will be employed to the Echinococcosis elimination. In addition, efficacy of treatment, vaccine, or deworming is not equal to community effectiveness. Many other significant factors, such as access rate, targeting accuracy, provider compliance, consumer adherence rate, and so on, all affect the final real control effects. For example, when carrying on deworming programme, dogs will recover partially but may be with very high re-infection rate. Also due to deworming programme, large quantity of eggs will be discharged into environment and consequently may cause many new infection to human and other hosts if the dog's droppings are not properly disposed. Investigating how these factors affect the control and elimination of Echinococcosis is essential for developing more successful strategies. Therefore, it is reasonable and realistic to explore what control strategies can be used to eliminate Echinococcosis in a shortest time (e.g., Before 2030). We leave this topic for the future investigation.

\section{Conclusions}

Our findings suggest that, the unknown amount of stray dogs and the use of sheep vaccination that with shorter effective-immunity duration can possibly lead to the abnormal growth of human and dog infections. Therefore, the optimal control measures must be carried out in real-world interventions of CE. It is reasonable and realistic to improve the effectiveness of vaccination and to reduce the food resources of stray dog, e.g., one can increase the effort for the pretest of sheep vaccination and reduce the richness of other wild intermediate hosts such as voles, Ochotona curzoniaes.

\section{Supplementary Information}

The online version contains supplementary material available at https://doi. org/10.1186/s40249-021-00807-6.

Additional file 1: Theoretical analyses.

Acknowledgements

We would like to thank anonymous reviewers for very helpful suggestions which improved greatly this manuscript.

\section{Authors' contributions}

$\mathrm{RX}, \mathrm{FM}, \mathrm{ZH}$ and $\mathrm{YH}$ participated in study conception and design, collection data; RX performed the mathematical analyses and numerical analyses; RX and FM wrote the manuscript. RX, FM and ZH carried out the initial analyses, reviewed and revised the manuscript; All authors contributed to the interpretation of the results, revised the manuscript critically, read and approved the final version of the manuscript.

\section{Funding}

The research was supported by National Natural Science Foundation of P. R. China (No. 12071068, 11671072), Natural Science Foundation of Jilin Province, P. R. China and partially supported by NSERC of Canada and York Research Chair Program.

\section{Availability of data and materials}

The data that support the findings of this study are available from the work $[11,12]$ and Sichuan Center for Disease Control and Prevention (https://www. sccdc.cn/View.aspx?id=012003).

\section{Ethics approval and consent to participate}

Not applicable.

\section{Consent for publication}

Not applicable.

\section{Competing interests}

The authors declare that there is no conflict of interests regarding the publication of this article. No authors have potential conflicts of interest with reference to this work.

\section{Author details \\ ${ }^{1}$ College of Mathematical Sciences, Harbin Engineering University, 145 Nan- tong Street, Harbin, Heilongjiang 150001, People's Republic of China. ${ }^{2}$ School of Mathematics and Statistics, Northeast Normal University, 5268 Renmin Street, Changchun, Jilin 130024, People's Republic of China. ${ }^{3}$ CDM, LAMPS}


and Department of Mathematics and Statistics, York University, 4700 Keele Street, Toronto, ON M3J 1P3, Canada. ${ }^{4}$ Animal Health Supervision Institute of Xingan League, Tiexi North Road, Ulanhot, Inner Mongolia 137400, People's Republic of China.

Received: 16 September 2020 Accepted: 9 February 2021 Published online: 24 March 2021

\section{References}

1. Craig PS, McManus DP, Lightowlers MW, Chabalgoity JA, Garcia HH, Gavidia CM, et al. Prevention and control of cystic echinococcosis. Lancet Infect Dis. 2007;7(6):385-94.

2. Budke CM. Global socioeconomic impact of cystic echinococcosis. Emerg Infect Dis. 2006;12:296-303.

3. Torgerson PR, Devleesschauwer B, Praet N, Speybroeck N, Willingham AL, Kasuga F, et al. The global burden of alveolar echinococcosis. PLoS Negl Trop. 2015;12(12):e1001920.

4. Torgerson PR, Schweiger A, Deplazes P, Pohar M, Reichen J, Ammann RW, et al. Alveolar echinococcosis: from a deadly disease to a well-controlled infection. Relative survival and economic analysis in Switzerland over the last 35 years. J Hepatol. 2008;49:72-7.

5. Torgerson PR, Keller K, Magnotta M, Ragland N. The global burden of alveolar echinococcosis. PLoS Negl Trop. 2010;4:e722.

6. Giraudoux P, Raoul F, Pleydell D, Li T, Han X, Qiu J, et al. Drivers of Echinococcus multilocularis transmission in China: small mammal diversity, landscape or climate? PLoS Negl Trop Dis. 2013;7(3):e2045.

7. Li TY, Chen X, Zhen R, Qiu J, Qiu D, Xiao N, et al. Widespread co-endemicity of human cystic and alveolar echinococcosis on the eastern Tibetan Plateau, northwest Sichuan/southeast Qinghai, China. Acta Tro. 2010;113:248-56.

8. Hartnack S, Budke CM, Craig PS, Jiamin Q, Boufana B, Campos-Ponce M, et al. Latent-class methods to evaluate diagnostics tests for Echinococcus infections in dogs. PLoS Negl Trop Dis. 2013;7:e2068.

9. Craig PS, Larrieu E. Control of cystic echinococcosis/hydatidosis: 1863-2002. Adv Parasitol. 2006;61:433-508.

10. Craig P, Hegglin D, Lightowlers M, Torgerson P, Wang Q. Chapter twoechinococcosis: control and prevention. Adv Parasitol. 2017;9:55-158.

11. He W, Wang Q, Huang Y, Huang L, Yu WJ, Yi DY. Analysis of the incidence of echinococosis (hydatidosis) in areas of Sichuan Province from 2007 to 2012. J Pathogen Biol. 2014;9(1):68-70 (in Chinese).

12. He W, Shang JY, Huanf Y, Wang Q, Yu WJ. Surveillance of hydatidosis in Sichuan. J Prev Med Inf. 2016;32(8):798-800 (in Chinese).

13. Sichuan Center for Disease Control and Prevention, Legal infectious disease epidemic cases. 2020. https://www.sccdc.cn/View.aspx?id=012003. Accessed 24 Aug 2020.

14. Budke CM, Qiu JM, Wang Q, Torgerson PP. Economic effects of echinococcosis in a disease-endemic region of the Tibetan Plateau. Am J Trop Med Hyg. 2005;73(41):2-10.

15. Yang YR, McManus DP, Huang Y, Heath DD. Echinococcus granulosus infection and options for control of cystic echinococcosis in Tibetan communities of Western Sichuan Province, China. PLoS Negl Trop Dis. 2009; 180:e426.

16. Moss JE, Chen X, Li T, Qiu J, Wang Q, Giraudoux P, et al. Widespread coendemicity of human cystic and alveolar echinococcosis on the eastern Tibetan Plateau, northwest Sichuan/southeast Qinghai, China. Parasitology. 2013;140:1685-92.

17. Lee S, Chowell G, Castillo-Chávez C. Optimal control for pandemic influenza: the role of limited antiviral treatment and isolation. J Theor Biol. 2010;265(2):136-50.

18. Blayneh KW, Gumel AB, Lenhart S, Clayton T. Backward bifurcation and optimal control in transmission dynamics of West Nile virus. Bull Math Biol. 2010;72(4):1006-28.
19. Zhao S, Kuang Y, Wu CH, Ben-Arieh D, Ramalho-Ortigao M, Bi K. Zoonotic visceral leishmaniasis transmission: modeling, backward bifurcation, and optimal control. J Math Biol. 2016;73(6-7):1525-60.

20. Cai L, Li X, Tuncer N, Martcheva M, Lashari AA. Global socioeconomic impact of cystic echinococcosis. Math Biosci. 2017;288:94-108.

21. Kato N, Kotani K, Ueno S, Matsuda H. Optimal risk management of human alveolar echinococcosis with vermifuge. J Theor Biol. 2010;267(3):265-71.

22. Rong XM, Fan M, Sun XD, Wang YM, Zhu HP. Impact of disposing stray dogs on risk assessment and control of Echinococcosis in Inner Mongolia. Math Biosci. 2018;2018(299):85-96.

23. Zhou MZ, Yang AG, Zhou ZX, Yuan DB, Guo L, Zhang CH, et al. Establishment and application of comprehensive treatment model for animal Echinococcosis at origin in Sichuan Province. Chin J Anim Health Inspect. 2017;34(7):32-5 (in Chinese).

24. Gauci C, Vural G, Oncel T, Varcasia A, Damian V. Vaccination with recombinant oncosphere antigens reduces the susceptibility of sheep to infection with Taenia multiceps. Int J Parasitol. 2008;38:1041-50.

25. Torgerson PR, Burtisurnov KK, Shaikenov BS, Rysmukhambetova AT, Abdybekova AM, Ussenbayev AE. Modelling the transmission dynamics of Echinococcus granulosus in sheep and cattle in Kazakhstan. Vet Parasitol. 2003; 114(2):143-53.

26. Yan S, Zhang Y. The control and prevention of livestock Echinococcosis in Xinjiang. Grass-Feeding Lives. 1994;180(4):45-7.

27. Fleming $W H$, Rishel RW. Deterministic and stochastic optimal controls. New York: Springer-Verlag; 1975

28. The People's Government of Sichuan Province, Input of prevention and control for Echinococcosis in Sichuan. 2020. http://www.sc.gov.cn/10462 /10464/10797/2018/9/30/10460060.shtml. Accessed 04 Nov 2020.

29. Lenhart S, Workman JT. Optimal control applied to biological models, mathematical and computational biology series. Boca Raton: Chapman \& Hall/CRC; 2007

30. den Van DP, Watmough J. Reproduction numbers and subthreshold endemic equilibria for compartmental models of disease transmission. Math Biosci. 2002;180(1):29-48.

31. Dietz K. Transmission and control of arbovirus diseases. In: Cooke KL, editor. Epidemiology. 1975. pp. 104-21.

32. Thieme HR. Convergence results and a Poincare-Bendixson trichotomy for asymptotically autonomous differential equations. J Math Biol. 1992;30(7):755-63.

33. Zhao XQ, Jing ZJ. Global asymptotic behavior in some cooperative systems of functional differential equations. Can Appl Math Q. 1996;4(4):421-44.

34. Healthy China 2030. 2020. http://www.gov.cn/xinwen/2019-07/15/conte nt_5409694.htm. Accessed 04 Nov 2020.

35. Sichuan Statistic Yearbook2008. 2020. http://tjj.sc.gov.cn/tjcbw/tjnj/2008/ chinese/dir/chinesemenu.htm. Accessed 24 Aug 2020.

36. WHO 2019 Sichuan Center for Disease Control and Prevention, Legal infectious disease epidemic cases. 2020. https://www.who.int/newsroom/fact-sheets/detail/echinococcosis. Accessed 24 Aug 2020.

37. Wang S, Ye S. Text book of medical microbiology and parasitology. Beijing: Science Press; 2006.

38. Torgerson PR, Heath DD. Transmission dynamics and control options for Echinococcus granulosus. Parasitology. 2003;127(S1):143-58.

39. LaSalle JP, Lefschetz S. Stability by Liapunov's direct method. 1. Cambridge: Academic Press; 1961.

40. Boltyanskii PV, Gamkrelize R, Mishchenko E. The mathematical theory of optimal processes. New York: Wiley; 1967.

41. Clayton TJ. Optimal control of epidemic models involving rabies and West Nile Viruses. Tennessee: University of Tennessee; 2008. 